\title{
Image Restoration Techniques: A Survey
}

\author{
Monika Maru \\ P. G. scholar \\ CSE Department \\ Gujarat Technological University, \\ Ahmedabad, India
}

\author{
M. C. Parikh, PhD \\ Associate Professor \\ CSE Department \\ Gujarat Technological University, \\ Ahmedabad, India
}

\begin{abstract}
During the process of image acquisition, sometimes images are degraded by various reasons. Image restoration is a challenging task in the field of Image processing. The process of recovering such degraded or corrupted image is called Image Restoration. Restoration process improves the appearance of the image. The degraded image is the convolution of the original image, degraded function, and additive noise. The process of restoration is deconvolved this degraded image to obtain noiselessly and deblurred original image. Various methods available for image restoration such as inverse filter, Weiner filter, constrained least square filter, blind deconvolution method etc. some of the methods are either linear or non-linear method helps to remove noise and blur from the image. In this description and comparison of restoration techniques are mentioned. In this paper, various spatial domain filters are discussed which are used to remove noise from the images.
\end{abstract}

\section{General Terms}

Algorithm

\section{Keywords}

Image Restoration, Degraded Image, Blur, Noise, PSF

\section{INTRODUCTION}

An image worth thousands of words and atmosphericturbulence affects the image quality so it is necessary to restore that degraded images. Generally, main causes of degradation are a blur, noise, and motion. Restoration of the image is a very big challenge in the field of image processing. To restore the image there must have knowledge of degradation. Restoration process improves the appearance of the image. Reconstruction of the image can be performed using 2 types of model (i) Degradation Model (ii) Restoration Model.

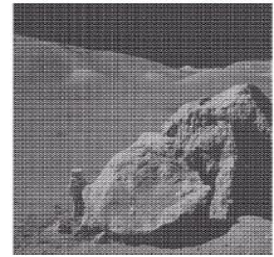

(a)
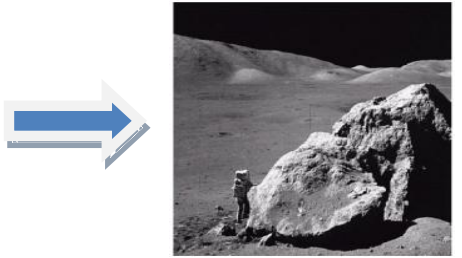

(b)
Figure 1(a) Degraded image (b) Restored image

In Figure 1 there are two images shown. Figure 1(a) is imperfect due to various reasons described in this paper. Figure 1(b) shows the clear image which is obtained by different types of restoration techniques. Generally, degradation caused at the moment of image acquisition and transformation of the image from one device to another device.

$$
g(x, y)=h(x, y) * f(x, y)+n(x, y)
$$

In above Equation (1) $f(\mathrm{x}, \mathrm{y})$ is an original image which is degraded by PSF (Point Spread Function) H. And once the image is degraded we need to restore it by adding noise $\mathrm{n}(\mathrm{x}$, y) and convolving restoration function. Finally, restored image $f^{\wedge}(x, y)$ is obtained. The Mathematical Equation (1) is represented as follows, where $\mathrm{h}(\mathrm{x}, \mathrm{y})$ is distortion. The symbol * represents convolution [2].

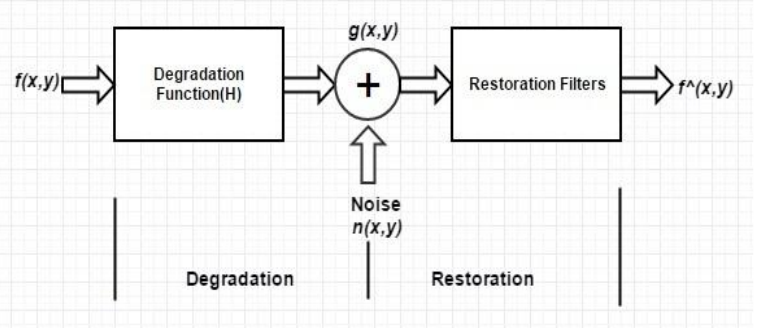

Figure 2 degradation-restoration Model

Restore the image using Non-blind restoration techniques and blind restoration techniques. Non-blind techniques further classified into linear restoration as well as Non-linear restoration techniques [4].

\subsection{Image Restoration vs. Image Enhancement}

Image restoration is different from Image Enhancement as Restoration is more Objective and Enhancement is Subjective. Image Enhancement could not precisely represent by mathematical function whereas in Image restoration is related to feature extraction from the imperfect image. Enhancement is manipulated the degraded image, increases the contrast of the image and visual appearance can be improved \{cite\}.

\subsection{Reasons for Occurrences of degradation}

There are many reasons available for degradation such as sensor noise, camera-misfocus, relative object-camera motion, random atmospheric turbulence. random variation of brightness or color information in the image is called noise it can be produced by sensor and circuitry of a scanner or digital camera [2]. While object moves to the camera or vice versa, motion blur can be caused [5]. While the object is out of focus of the camera during exposure, the object region in the image is also blurred. This kind of blur is called defocus blur [6] imaging system is affected by atmospheric turbulence by virtue of wave propagation through a medium with nonuniform index of refraction.

The situation in which degradation occurs is image acquisition and transmission of the image [4]. Types of distortions are 
space variant and space invariant where all pixels are suffered from the same distortion problem is space invariant degradation and the distortion suffered by pixels in the image are depending upon their location called space invariant [7]. Space invariant occurred due to camera motion or global lack of focus. Space-variant distortion is complex as it depends on their location with compare to space invariant [8].

\section{NOISE MODEL}

Production factor of noise in images is image acquisition, transmission as well as low/high level of lights, atmospheric problem and sensor temperature [9]. Noise operates by restoration model in spatial domain where playing with pixels directly [3].

\subsection{Gaussian Noise}

It has a random distribution of amplitude over time and it occurs frequently and known as normal noise. Probability density function of Gaussian noise is:

$$
p(z)=\frac{1}{\sqrt{2 \pi \sigma^{2}}} e^{-(z-\mu)^{2}} / 2 \sigma^{2}
$$

Where $\sigma$ is a standard deviation and $\mathrm{z}$ is intensity value [3].

\subsection{Uniform Noise}

During Uniform Noise, Pixels of sensed image Quantized to a number of discrete levels. That is why also called quantization noise.

$$
p(z)=\left\{\begin{array}{cl}
\frac{1}{b-a} & \text { if } a \leq z \leq b \\
0 & \text { otherwise }
\end{array}\right.
$$

Mean and variance of uniform noise is:

$$
\begin{aligned}
& z=a+b / 2 \\
& \sigma^{2}=\frac{(b-a)^{2}}{2}
\end{aligned}
$$

\subsection{Impulse Noise}

It is the combination of white and black dots and also called salt-pepper noise.

$$
p(z)=\left\{\begin{array}{cl}
P a, & \text { if } z=a, \\
P b & \text { if } z=b, \\
0 & \text { otherwise }
\end{array}\right.
$$

Where intensity of $b$ is white dots means salt, where $b>a$ in image and intensity of a is black dots means pepper where $a>b$ in image [3].

\section{BLUR MODELS}

Sometimes blur may be produced by the photographer to strengthen photo's expressiveness, but unintentional blur will decrease the image quality, which is caused by incorrect focus, object motion, hand shaking and so on [10]. Convolution of the ideal image with 2-D point-spread function (PSF) $n(x, y)$ in equation (1) is blurring of the image. Different types of blurs are also responsible for degradation; blurred images are restored by restoration model in the frequency domain.

\subsection{Average Blur}

It uses to remove noise and specks from the image. it used when noise is present over the entire image. Paper Average blurring can be distributed in horizontal and vertical direction and can be finding by circular averaging of radius $\mathrm{R}$ which is evaluated as:

$$
\mathrm{R}=\text { square root of } \sqrt{g^{2}}+f^{2}
$$

where $\mathrm{g}$ is the horizontal size blurring direction and $\mathrm{f}$ is vertical blurring size direction and $\mathrm{R}$ is the radius size of the circular average blurring [11].

\subsection{Gaussian blur}

It is a filter that blends a specific number of pixels incrementally, following a bell-shaped curve. Blurring is dense in the center and feathers at the edge [11]. In remote sensing, atmospheric turbulence is a severe limitation. The occurrence of Gaussian blur depends on a variety of factors such as temperature, wind speed, exposure time [12]. The Gaussian Blur effect is a filter that blends a specific number of pixels incrementally, following a bell-shaped curve [11].

\subsection{Motion blur}

There is relative motion between camera and object due to the image capturing called motion blur. Many types of motion blur can be distinguished all of which are due to relative motion between the recording device and the scene. This can be in the form of a translation, a rotation, a sudden change of scale, or some combinations of these. Here only the important case of a global translation will be considered.

\subsection{Defocus blur}

The situation in which object is going out of focus of lenses due to image capturing called Defocus blur. When a camera images a 3-D scene onto a 2-D imaging plane, some parts of the scene are in focus while other parts are not. If the aperture of the camera is circular, the image of any point source is a small disk, known as the circle of confusion (COC). The degree of defocus (diameter of the COC) depends on the focal length and the aperture number of the lens, and the distance between camera and object. An accurate model not only describes the diameter of the COC, but also the intensity distribution within the COC [11] [13].

\section{RESTORATION TECHNIQUES}

There are various restoration techniques as well as spatial domain filter for noise removal. In spatial domain methods, the technique operates directly on the pixels of an image. The spatial domain methods are used for removing additive noise only. Sometimes blur helps to increase photo's expressiveness but it decreases the quality of image unintentionally. In image restoration, the improvement in the quality of the restored image over the recorded blurred one is measured by the signal-to-noise ratio improvement [14]. Image restoration techniques are used to make the corrupted image as similar as that of the original image. Figure. 3 shows classification of restoration techniques. Basically, restoration techniques are classified into blind restoration techniques and non-blind restoration techniques [15]. Non-blind restoration techniques are further divided into linear restoration methods and nonlinear restoration method [16]. 


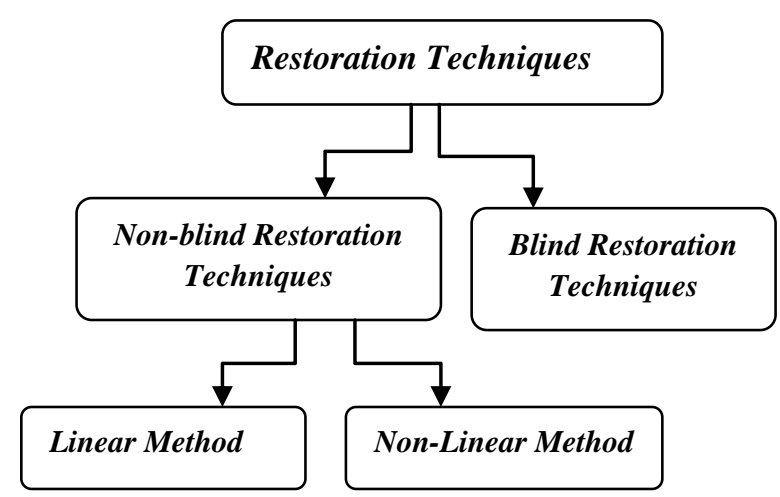

Figure 3 Classification of restoration techniques

Non-blind restoration techniques are described first in this paper. A non-blind technique depends on the estimation of PSF which should be priorly known. Based on PSF estimation it restores the input image. As mentioned above other two types of non-blind techniques are linear restoration methods such as Weiner filter, Inverse filter, and Constrained Least square filter. Lucy-Richardson algorithm is a Non-linear type of restoration method [7]

\subsection{Direct Inverse Filtering}

The blurring function of the corrupted image is known or can be developed then it has been proved as quickest and easiest way to restore the distorted image. Blurring can be considered as low pass filtering in inverse filtering approach and use high pass filtering action to reconstruct the blurred image without much effort. Suppose first that the additive noise is negligible. A problem arises if it becomes very small or zero for some point or for a whole region in the plane then in that region inverse filtering cannot be applied.

\subsection{Wiener Filter}

This is a standard image restoration approach proposed by $\mathrm{N}$. Wiener that incorporates both the degradation function and statistical characteristic of noise into the restoration function. It is one of the best deblurring linear methods which reconstructs an image from degraded image by using known PSF. It works with high pass filter to perform Deconvolution as well as with low-pass filter to remove noise with compression operation.

$$
f=g \times(f+n)
$$

The output image is obtained by above equation where additive noise and frequency characteristics are a known[2] [11].It compares with an estimation of the noiseless image which is wanted or desired. The input to a wiener filter is a degraded image corrupted by additive noise.

\subsection{Constraint Least-Square Filter}

Regularized filtering is used in a better way when constraints like smoothness are applied on the recovered image and very less information is known about the additive noise. The blurred and noisy image is regained by a constrained least square restoration algorithm that uses a regularized filter. Regularized restoration provides almost similar results as the wiener filtering but viewpoint of both the filtering techniques are different. In regularized filtering less previous information is required to apply restoration. The regularization filter is frequently chosen to be a discrete Laplacian. This filter can be understood as an approximation of a Weiner filter.

The second name of filter called Regularized filter is a vectormatrix form of linear degradation model. Equation is [17]:

$$
g=H f+n
$$

Where $\mathrm{f}$ is $\mathrm{M}^{*} \mathrm{~N}$ vector whose first $\mathrm{N}$ elements are first-row pixels of the image, next $\mathrm{N}$ elements are second-row pixels up to $\mathrm{MN} * \mathrm{MN}$. Now the problem is simple matrix multiplication so now need to find the second deviation of degraded image [4].

Now, a blind restoration technique is discussed. In order to overcome the problems of typical image restoration methods' being confined the large estimation error of PSF and the complex computation in blind image restoration methods. Blind deconvolution is a deconvolution technique that permits recovery of the target scene from a single or set of "blurred" images in the presence of a poorly determined or unknown point spread function (PSF). Regular linear and non-linear deconvolution techniques utilize a known PSF. For blind deconvolution, the PSF is estimated from the image or image set, allowing the deconvolution to be performed.

Spatial domain filters are categorized as Mean Filters, Order Statistic Filter, and Adaptive Filter. The filters are used to remove noise and disturbances from the images. The Spatial domain filters are used to operate directly on the pixels of an image, whereas the Frequency domain filters operate on intensities of an image. The spatial domain filters are very efficient. They require less number of resources. Such as a simple filter mask for a filter effect, So it is less expensive to perform filtering in the spatial domain. Frequency domain filtering is more appropriate if no straight forward or simple mask can be found in the spatial domain.

\subsection{Mean Filters}

\subsubsection{Arithmetic Mean Filters}

The Arithmetic mean filter is also called Linear Filter. It averages all the values of pixels within the window. The arithmetic filter is the simplest form of the mean filter. This filter helps in smoothing the variations in an image and it blurs the image. It normally blurs the edges. This may be a problem if sharp edges are required in the desired output.

\subsubsection{Geometric mean filter}

Same as an arithmetic mean filter but it loses less image detail when processing the image. Filter size is large so it is giving blurring effect.

\subsubsection{Harmonic mean filter}

It is used in a situation in which data values are so high. it cannot denoise the pepper noise. This is the best filter for gaussian noise and salt noise.

\subsubsection{Contra harmonic mean filter}

This filter is best for eliminating salt and pepper noise. It can't remove both the noises at the same time. If choose wrong values then the filter can behave as a dragon. The Fig A and Fig B shows the negative impact of this filter.

The filter removes pepper noise and for its negative value, it destroys salt noise. If choose a wrong value then filter gives the worst results. 

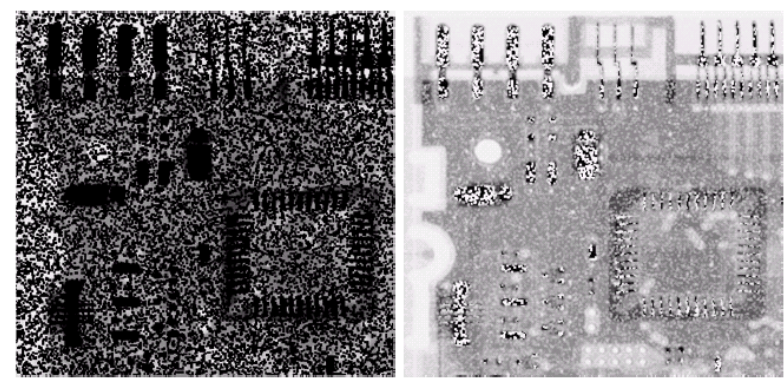

Fig (A) pepper noise filtered by $Q=-1.5$ Fig (B) Salt noise filtered by $Q=+1$.

\subsection{Order Statistic Filter}

In these type of filters, the values of the pixels of an image are ranked in order. Only those pixel values are ranked whose area or region is enclosed in the filter.

\subsubsection{Median Filter}

This filter first calculates the median of the intensity levels of the pixels Suppose the pixel values are from 1-9, so the median will be 5 , that is, the midpoint of the pixel values. Then after calculating the median, it replaces the corrupted pixel value with the new value (median value).

This filter is more robust because single pixel in the neighborhood never affects median value. It is much better at preserving sharp edges than another filter. But it is more expensive and complex to execute. It is taking much time to calculate a median value for each window.

\subsubsection{Max and Min Filter}

These filters are used to find the brightest and darkest points in the image. The Max filter replaces the pixel value with the brightest point and the Min filter replaces the pixel with the darkest point. Max filter helps to find light colored pixels in an image while Min filter helps to find dark points in the image.

\subsubsection{Midpoint Filter}

The Midpoint filter computes the midpoint between the maximum and minimum values of the image. The midpoint filter is widely used for noises like Gaussian noise and uniform noise. but it works well only for randomly distributed noise.

\subsubsection{Alpha-trimmed Mean Filter}

As the name implies, this filter trims the $d / 2$ highest and $d / 2$ lowest intensity values of the corrupted image in $S_{x y}$. Let $\operatorname{gr}(\mathrm{s}, \mathrm{t})$ represents remaining mn-d pixels. Then the alphatrimmed mean filter averages the value of these remaining pixels. The value of $\mathrm{d}$ ranges from 0 to $\mathrm{mn}-1$. When $\mathrm{d}=0$, then alpha-trimmed filter becomes arithmetic mean filter. If $\mathrm{d}=\mathrm{mn}-1$ then, the filter becomes a median filter. It is useful in a situation such as multiple types of noise, Gaussian noise, and the combination of salt and pepper noise.

\subsection{Adaptive Mean Filter}

This filter is the third type of spatial domain filters. In Adaptive median filters, the size of the filter can be change. The other filters discussed above can only be used for the images where the density of the noise is less. But this filter is used especially to remove high-density noise from corrupted images.

Table 1. Comparison table of restoration methods

\begin{tabular}{|c|c|c|c|c|c|c|}
\hline Techniques & $\begin{array}{c}\text { Salt-pepper } \\
\text { noise }\end{array}$ & $\begin{array}{c}\text { Gaussian } \\
\text { noise }\end{array}$ & $\begin{array}{c}\text { Uniform } \\
\text { noise }\end{array}$ & Motion blur & Defocus blur & $\begin{array}{c}\begin{array}{c}\text { Gaussian } \\
\text { blur }\end{array} \\
\end{array}$ \\
\hline Direct Inverse Filter & No & No & No & Yes & Yes & Yes \\
\hline Wiener Filter & Yes & Yes & Yes & Yes & Yes & Yes \\
\hline $\begin{array}{l}\text { Constrained least-Square } \\
\text { Filter }\end{array}$ & - & - & - & Yes & Yes & Yes \\
\hline Arithmetic Mean Filter & No & Yes & Yes & No & No & No \\
\hline Geometric Mean Filter & Yes & Yes & No & No & No & No \\
\hline Harmonic Mean Filter & $\begin{array}{l}\text { Salt-Yes, } \\
\text { Pepper-No }\end{array}$ & Yes & No & No & No & No \\
\hline $\begin{array}{c}\text { Contra-harmonic Mean } \\
\text { Filter }\end{array}$ & Yes & No & No & - & - & - \\
\hline Median Filter & Yes & Yes & No & Yes & Yes & No \\
\hline Max and Min Filter & Yes & No & No & No & No & No \\
\hline Midpoint Filter & No & Yes & Yes & No & No & No \\
\hline Adaptive Mean Filter & Yes & No & No & No & No & No \\
\hline
\end{tabular}

\section{APPLICATION}

The first application of digital image restoration in the engineering community was in the area of astronomical

characterized by Poisson noise, Gaussian noise. In the area of imaging, image restoration has played a very important role. astronomical imaging degradation problem is often medical
Restoration has been used for mammograms, filtering of camera shutter speeds relative to rapid spacecraft motion [18]. The Poisson distributed film grain noise in chest X-rays and digital planets were degraded by motion blur as a result of slow angiographic images, and for the removal of additive noise in imaging [19]. Extra terrestrial observation of the earth and the Magnetic resonance Imaging. 


\section{CONCLUSION}

Restoration techniques are used to restore corrupted image into its original form. Restoration Techniques improves the image quality. In this paper definition and objectives of image restoration are addressed. In this paper noise model and blur model, blurring and deblurring techniques are elaborated and its merits and demerits are explored. This discussion is useful for researcher to identify the restoration techniques based on noise and blur model.

\section{REFERENCES}

[1] B. Xiao-Jun and W. Ting, "Adaptive blind image restoration algorithm of degraded image," Proc. - 1st Int. Congr. Image Signal Process. CISP 2008, vol. 3, pp. 536-540, 2008.

[2] T. Singh, "Novel Restoration Process for Degraded Image," in 2014 Fifth International Conference on Signal and Image Processing, 2014, pp. 81-86.

[3] R. Kaur and E. N. Singh, "Image Restoration - A Survey," IOSR J. Comput. Eng., vol. 16, no. 4, pp. 107111, 2014.

[4] S. Kumar, "Performance Evaluation and Analysis of Image Restoration Technique using DWT," vol. 72, no. 18, pp. 11-20, 2013

[5] C. Liu, Y. Zhang, H. Wang, and X. Wang, "Improved block Kalman filter for degraded image restoration," Proc. - 2013 IEEE Int. Conf. High Perform. Comput. Commun. HPCC 20132013 IEEE Int. Conf. Embed. Ubiquitous Comput. EUC 2013, pp. 1958-1962, 2014.

[6] D. Yang and S. Qin, "Restoration of degraded image with partial blurred regions based on blur detection and classification," in 2015 IEEE International Conference on Mechatronics and Automation (ICMA), 2015, pp. 2414-2419.

[7] A. Thakur and A. Iqbae, "Comparison efficacy of restoration method for Space Variant Motion Blurred Images using kalman \& wiener filter," 2016.

[8] W. F. Al Maki, T. Shimahashi, T. Kitagawa, and S. Sugimoto, "Space invariant blur estimation and noiseless kalman filter-based image deconvolution," Int. J. Innov. Comput. Inf. Control, vol. 5, no. 1, pp. 201-213, 2009.

[9] M. Aggarwal, R. Kaur, and B. Kaur, "A Review of Denoising Filters in Image Restoration," Int. J. Curr.
Res. Acad. Rev., vol. 2, no. 3, pp. 83-89, 2014.

[10] B. Su and S. Lu, "Blurred Image Region Detection and Classification.'

[11] A. Maurya and R. Tiwari, “A Novel Method of Image Restoration by using Different Types of Filtering Techniques," Int. J. Eng. Sci. Innov. Technol., vol. 3, no. 4, pp. 124-129, 2014.

[12] D. Broutman, J. W. Rottman, and S. D. Eckermann, "R Ay M Ethods for I Nternal W Aves in the," pp. 1-25, 2004.

[13] P. Dhole and N. Chopde, "A Comparative Approach for Analysis of Image Restoration using Image Deblurring Techniques," Int. J. Curr. Eng. Technol., vol. 5, no. 2, pp. 1046-1049, 2015.

[14] R. Nagayasu, N. Hosoda, N. Tanabe, H. Matsue, and T. Furukawa, "Restoration method for degraded images using two-dimensional block Kalman filter with colored driving source," 2011 Digit. Signal Process. Signal Process. Educ. Meet. DSP/SPE 2011 - Proc., pp. 151156, 2011.

[15] A. Thakur, A. Kausar, and A. Iqbal, "Comparison efficacy of restoration method for space variant motion blurred images using kalman \&amp; wiener filter," in 2016 6th International Conference - Cloud System and Big Data Engineering (Confluence), 2016, pp. 508-512.

[16] P. D. Samarasinghe and R. A. Kennedy, "On Non-blind Image Restoration," 2009.

[17] Chidananda Murthy M V, M. Z. Kurian, and H. S. Guruprasad, "Performance evaluation of image restoration methods for comparative analysis with and without noise," in 2015 International Conference on Emerging Research in Electronics, Computer Science and Technology (ICERECT), 2015, pp. 282-287.

[18] Y. Wang, "Image filtering: noise removal, sharpening, deblurring," http//eeweb.poly.edu/ yao Polytech., p. 41, 2006.

[19] L. Yan, M. Jin, H. Fang, H. Liu, and T. Zhang, "Atmospheric-turbulence-degraded astronomical image restoration by minimizing second-order central moment," IEEE Geosci. Remote Sens. Lett., vol. 9, no. 4, pp. 672676, 2012. 